\title{
Exploratory research on GPS measuring technique in practice of engineering surveying
}

\author{
Fei Liu \\ Changjiang Institute of Technology, Wuhan, 430212, China
}

Keywords: GPS measuring technique; Engineering surveying; Problems and Countermeasures

\begin{abstract}
With development of science and technology, GPS measuring technique also makes some progress nowadays and plays an increasingly important role in practice of engineering surveying. Although GPS measuring technique has many advantages, such as high technical content, high measurement accuracy and short measuring time, it still has some problems in practical application, which we need pay attention to. This thesis simply analyzes composition, technical advantages and application of GPS measuring technique, focuses on studying problems of GPS in engineering practice and proposes solutions via exploration.
\end{abstract}

\section{Introduction}

GPS just refers to global positioning system, which belongs to radio navigation positioning system in essence. It is jointly composed of global communication satellite and signal reception devices and can provide accurate navigation, time information and three-dimensional coordinate for users. As the latest satellite positioning navigation system, GPS can not only realize all-weather, global and uninterrupted three-dimensional navigation positioning function but also have strong confidentiality and anti-interference performance. As global digitization process accelerates, GPS technique develops rapidly, is widely applied to engineering surveying and plays an important role.

\section{Composition and advantages of GPS system}

\section{Composition of GPS system}

Global positioning system (GPS) mainly contains two sub-systems, i.e., ground monitoring system and space satellite group system. According to hardware facilities, it also contains satellite receiving equipment. The space satellite group includes 24 satellites in all and all satellites are averagely distributed to 6 orbits surrounding the earth, whose distance to the ground is 0.2 million kilometers. On such orbits, operational cycle of satellites is 11 hours and 58 minutes. In this way, any site on the earth can receive GPS signals sent by 4 satellites at least and 11 satellites at most simultaneously anytime. Devices contained by GPS user terminal part involve receiver, data processing equipment, corresponding user equipment, meteorological instrument and computer etc. Via receiving signals sent by satellites, specific position coordinates can be obtained according to such signals.

\section{Advantages of GPS system}

The reason why GPS is widely applied to many fields is that is has powerful functional advantages. Such advantages are mainly reflected by three aspects, including high positioning accuracy, all-weather uninterrupted work and simple and easy operation. First of all, GPS has extremely high positioning accuracy. Satellite navigation technique is the main technology that GPS measuring technique relies on in practical application. In the aspect of height, satellites have some advantages. When objects that need detection are detected by satellites, we may have more comprehensive observation. Meanwhile, when GPS measuring technique measures objects, it can define ranges of objects accurately. Thus, its detection accuracy is quite high, which provides advantages for final measuring results with high accuracy and promotes improvement in accuracy of measuring technique as well. Thus, compared with other measuring techniques, GPS measuring technique has quite high measurement accuracy. Secondly, GPS is featured by all-weather operation. When other measuring techniques carry out engineering surveying, they are usually affected by 
weather and other external factors. However, GPS measuring technique is different. When it implements measurement and observation, changes in weather will not affect its measurement so that it can implement all-weather and uninterrupted measurement. This not only improves work efficiency but also avoid various kinds of interference problems in measurement. Some traditional engineering surveying techniques rely on manual operation of related workers largely in practical measurement, so measurement must be stopped once weather changes rapidly or heavy rain or heavy snowfall comes in measurement. Such a situation has impacts on successful implementation of engineering. However, application of GPS measuring technique can avoid such problems completely and shorten time of engineering measurement. Thirdly, GPS technique has simple operation and strong practicability. In accordance with the whole process of GPS application, its operational links are not complicated. In detail, measuring instruments are used to analyze measured objects and obtain data finally in the process of measurement. The process of measurement is mainly divided into three steps. At the first step, decided target objects of measurement. Before measurement, it is essential to adjust measuring positions and help GPS system find target objects of measurement. This can ensure normal measurement. After objects of measurement have been decided, set various kinds of measurement parameters. At this moment, measuring technique will construct a fuzzy model according to situations of objects. The model can help GPS technique analyze and compute measured data accurately when practical measurement is carried out. The second step involves data pre-processing. Since GPS technique can offer all-weather and uninterrupted work, it can give rapid analysis after obtaining metrical data and record data in time simultaneously.

\section{Application of GPS measuring technique to practice of engineering surveying}

Application of GPS measuring technique to practice of engineering surveying mainly includes three aspects, i.e., application of GPS positioning technique, measurement on construction benchmark and application of virtual reality technique. Firstly, GPS positioning technique has wide application to practice of engineering surveying, and its basic principle contains physics-related principle and geometry-related principle, combines principles of two different subjects, implements multi-angle measurement for objects by space satellites and lets ground receiving devices receive signals sent by satellites. Main application of GPS positioning technique to practice of engineering surveying contains static relative positioning and dynamic relative positioning. Operation of the static relative positioning is simple and its process it not complicated. In detail, receiving devices on the ground are arranged in the form of a base line to perform synchronous measurement on objects for about 45 minutes and then specific workers implement statistics and processing for measured data. Relative view on carrier waves is main theoretical basis of dynamic relative positioning measurement. Specifically, when measurement is carried out, a relatively accurate position on the ground is selected as a control base station of measurement and then several receiving devices are installed to give real-time observation on measured objects from several angles. Generally, GPS contains 24 satellites surrounding the earth in all. If an observation point is planed at an angle above $10^{\circ}$, signals of 7 satellites will be received in all. If there are buildings around receiving devices and signal transfer is affected, the number of satellites whose signals that receiving devices can receive will be smaller and the positioning difficulty of receivers will be increased. Thus, to obtain accurate measurement results under special situations, GPS positioning technique should implement measurement together with inertial navigation technique. Secondly, measurement on construction benchmark. Under normal conditions, design units usually use traditional measuring techniques when they measure engineering benchmarks. However, traditional measuring technique has some the problem that they cannot give rigorous budget or field investigation and the distance between two benchmarks usually tend to be larger. The distance between two benchmarks, which is provided by design units, usually ranges from $500 \mathrm{~m}$ to $1,000 \mathrm{~m}$. Such a large distance seriously affects normal progress of construction and delays days for construction. Benchmarks measured by the satellite signals that receiving devices of GPS system obtain can coordinate with progress of engineering observation well, improve accuracy of engineering measured data and shorten work practice. When 
GPS measurement technique measures benchmarks of construction, its basic process is shown as follows: installing receiving antenna, operating receiving devices and observing measuring records. In practical measurement, it is essential to carry out measurement according to measurement plans that have been made in advance and rules in such plans. Thirdly, application of virtual reality technology. In traditional measurement, development of measurement must rely on professional survey crews to implement field observation at construction sites. If weather conditions at construction sites are bad or geological conditions are poor, accidents will be caused easily. GPS virtual reality technology can create environment with reality and interaction and solve such problems completely. Based on GPS virtual reality technology, use computer drawing technique to draw three-dimensional images with high definition, establish specific steps of engineering surveying and mapping and then know key content and measurement problems that we must pay attention to in measurement. Engineering surveying and mapping can simulate specific processes of measurement, which not only overcomes the difficulty that surveying and mapping efficiency is low in actual measurement effectively but also enhances operability and safety of measurement plans.

\section{Research on problems of GPS measuring technique in practice of engineering surveying and countermeasures}

\section{Research on problems about measuring sites' setting and base stations' positioning and countermeasures}

First of all, when primary control network is set, setting of measuring sites is of great significance. GPS measuring sites should locate at the section where elevating angle of obstacles around base stations does not exceed $10^{\circ}$ and there is wide view. At the same time, objects that will reflect GPS signals should be avoided around measuring sites to prevent multipath errors from appearing. In addition, it is essential to create the condition that is helpful for transfer of differential correction signals for measuring sites. When position of measuring sites is selected, places with open space should be selected and we must ensure there are no continuous obstacles in the range of $10^{\circ}-15^{\circ}$ elevating angle. It is essential to prevent electromagnetic wave interference sources from appearing within $200 \mathrm{~m}$ whose center is a measuring site, such as high-power wireless launcher and high voltage transmission line and keep electromagnetic waves produced by such devices from interfering with satellite signals.

Secondly, when positioning of construction drawing is implemented via PTK, it will be necessary to re-position the site where the base station is placed if placement of the base station at a given point is unreasonable. Usually, multipoint positioning is used to position an appropriate place for placement of the base station. We may measure the place where the base station is placed by the following methods: WGS-84 coordinates of give points of two moving stations are measured rapidly'; according to measuring principle, actual coordinates of the base station are measured; and related parameters of independent coordinates are solved in accordance with obtained coordinate positions. If the base station is set by such methods, disturbing influence of signals sent by broadcasting stations on the base station will be reduced largely and accuracy of positioning will be higher.

\section{Research on problems about selection of GPS benchmark and baseline solution and countermeasures}

First of all, when GPS positioning achievement are applied to coordinate system, we must measure existing GPS points and GPS network together and the total number of jointly measured points must not be less than 2. Surveying and mapping accuracy of GPS network will be directly affect by distribution of points and errors. When the benchmark has large errors, system errors will appear in residual errors of surveying and mappings, which will cause deformation for GPS positioning results finally. Thus, selecting GPS network's benchmark accurately is the most important link in the process of GPS data processing. Currently, only Level A and Level B GPS network with high accuracy has been established in China. Since point location density fails to reach requirements of high-accuracy survey, it is not widely applied at present. When GPS technique is used for measurement in practice of engineering surveying, joint measurement can be 
carried out for coordinate transforming direction, dimension and location base according to the country acting as a large point. Secondly, problems in baseline solution and countermeasures. In baseline solution, the following 5 problems usually exist. The first problem is that baseline starting coordinates are not accurate enough. Aiming at the problem, we should choose points with higher accuracy or derive baseline starting coordinates from the same point uniformly when a solution starting point is selected. In this way, the obtained baseline results have errors. Then, use system parameter method to process balancing of GPS network. Secondly, there are excessive cycle slips. If only a satellite usually encounters the phenomenon of cycle slips, we only need delete observed values of the satellite. If several satellites suffer cycle slips in a certain period, we should delete all measured results in the period. In doing so, solution results can be ensured.

Thirdly, observation time of GPS satellites is not long enough. When baseline solution is implemented, we should delete observation data of a satellite if its observation time is too short to improve quality of solution results. Fourthly, multipath effectiveness is relatively serious. If multipath effectiveness exists, residual errors of observation data will be large. At this moment, we may delete observation data in a certain period or of a certain satellite to improve accuracy of solution results. Fifthly, impacts of troposphere and ionospheric refraction are too significant. At this time, we may delete data about impacts of troposphere and ionized stratum. Besides, the problems falsity exists in application of GPS measuring technique to practice of engineering surveying. Fixed solution and floating point solution will appear after failure in initialization. High measurement accuracy can be ensured only when RTK measurement of GPS instruments is used. However, being affected by processing software and various environmental factors, receivers tend to suffer deviation of plane position in the process of initialization. To prevent appearance of falsity, the following countermeasures are adopted generally: after re-initialization, two given points are re-measured; in operation process, observed elevation values are compared with actual numerical values to judge whether elevation values have abnormal conditions or not; in case of abnormal conditions, we need re-measure pints with such conditions and then carry out indoor processing.

\section{Conclusion}

All in all, GPS measuring technique has obvious technical advantages. Compared with traditional measuring techniques, it can improve accuracy and efficiency of measurement to a larger extent, avoid measurement workers' field observation and ensures safe implementation of measurement. Although GPS measuring technique has some problems in the process of application, which we need pay attention to, it is no doubt that effect of GPS technique can be completely exerted finally as long as we implement constant practice, summarization and exploration.

\section{Reference:}

[1] Wu Yingjie. Experience about GPS in engineering surveying [J]. Municipal Engineering of China, 2005(2).

[2] Li Jun. GPS measuring technique and its application to engineering surveying [J]. Journal of Qiqihaer Vocational College, 2012(5).

[3] Ma Jie. Discussion on improvement in accuracy and reliability of GPS RTK landmarks [J]. Zhongzhou Coal, 2006(2). 